\title{
Autorrelatos do amor no ciclo vital adulto
}

\author{
Self-reports of love in life cycle adult
}

Autoinformes de amor en el ciclo de la vida adulta

\author{
José Augusto Evangelho Hernandez* \\ Universidade do Estado do Rio de Janeiro - UERJ, Rio de Janeiro, Rio de Janeiro, \\ Brasil
}

\section{Sinele Valle da Costa**}

Universidade do Estado do Rio de Janeiro - UERJ, Rio de Janeiro, Rio de Janeiro, Brasil

\section{Juliana Ramos Ribeiro***}

Universidade do Estado do Rio de Janeiro - UERJ, Rio de Janeiro, Rio de Janeiro, Brasil

\section{Caroline Almeida Areias****}

Universidade do Estado do Rio de Janeiro - UERJ, Rio de Janeiro, Rio de Janeiro, Brasil

\section{Karina Nascimento Valladares dos Santos $* * * * *$}

Universidade do Estado do Rio de Janeiro - UERJ, Rio de Janeiro, Rio de Janeiro, Brasil

\begin{abstract}
RESUMO
Esta pesquisa transversal comparou grupos de pessoas de diferentes faixas etárias, adultos jovens, maduros e maiores de 50 anos. Foram verificadas possíveis modificações do amor ao longo do ciclo vital adulto. Participaram 211 sujeitos de ambos os sexos, envolvidos em tipos variados de relações amorosas, que responderam à Escala Triangular do Amor de Sternberg. Os resultados da análise de variância multivariada mostraram interações significativas entre os componentes do amor e as faixas etárias e entre os componentes do amor e o sexo dos participantes. Análises univariadas revelaram diferenças significativas em intimidade entre as faixas etárias. Além disso, os níveis de paixão, intimidade e compromisso dos homens foram mais elevados do que os das mulheres. Estes resultados foram discutidos à luz da literatura que aborda a Teoria Triangular do Amor.

Palavras-chave: psicologia do amor, paixão, intimidade, compromisso.
\end{abstract}

\section{ABSTRACT}

This cross-sectional study compared groups of people of different ages, young, mature and old adults. Possible modifications of love throughout the adult life cycle were observed. Participated in 211 subjects of both sexes, 
who were involved in various types of love relationships and answered Sternberg's Triangular Love Scale. The results of variance multivariate analysis showed significant interactions between the components of love and age groups and between the components of love and sex of the participants. Univariate analysis revealed significant differences in intimacy between age groups. In addition, the levels of passion, intimacy and commitment of men were higher than those of women. These results were discussed by the literature that addresses the Triangular Theory of Love.

Keywords: psychology of love, passion, intimacy, commitment.

\begin{abstract}
RESUMEN
Este estudio transversal comparó grupos de personas de diferentes edades, los adultos jóvenes, maduros y viejos. Se observaron posibles modificaciones de amor en todo el ciclo de vida del adulto. Participaron en 211 sujetos de ambos sexos, que estaban involucrados en los distintos tipos de relaciones amorosas y contestaron la Escala Triangular del Amor de Sternberg. Los resultados del análisis de varianza multivariado mostraron interacciones significativas entre los componentes del amor y grupos de edad y entre los componentes del amor y el sexo de los participantes. Los análisis univariados revelaron diferencias significativas en la intimidad entre los grupos de edad. Además, los niveles de pasión, intimidad y el compromiso de los hombres fueron más altos que los de las mujeres. Estos resultados fueron discutidos por la literatura que aborda la Teoría Triangular del Amor.
\end{abstract}

Palabras clave: psicología del amor, pasión, intimidad, compromiso.

\title{
1 Introdução
}

Em adolescentes, a crise psicossocial caracteriza-se pelos sentimentos opostos de intimidade versus isolamento. $O$ jovem quer um relacionamento, não quer permanecer isolado, está ansioso para compartilhar sua intimidade, entretanto teme não ser capaz de estabelecer um relacionamento profundo. $\mathrm{Na}$ medida em que se desenvolve a solução para essa contradição, poderá emergir o amor. $\mathrm{O}$ adulto jovem já não tem as mesmas mudanças de humor e os conflitos freqüentes, característicos dos adolescentes. Em torno dos vinte anos de idade, inicia a separação das famílias de origem e a preparação para a vida nas dimensões acadêmicas, profissionais e sociais (Erikson, 1998).

Entre a terceira e quinta décadas da vida, as pessoas se esforçam, principalmente para consolidar suas escolhas profissionais. Além disso, assistem à sua família nuclear e de origem, vivenciam o nascimento, o crescimento e o empenho dos filhos na conquista da independência (Smetana, Campione-Barr, \& Metzger, 2006) e no enfrentamento das dificuldades da vida (Arnett, 2000). A meia idade também é caracterizada pelo declínio do vigor, da força, da juventude, dos sonhos e das aspirações irrealistas.

As mudanças fisiológicas que ocorrem durante a vida adulta sugerem que adultos mais velhos relatem níveis mais baixos de paixão do que 
os mais jovens. A maioria dos estudos mostra que a paixão se torna menos proeminente, contudo, esta diminuição da paixão é pequena (Ahmetoglu, Swami, \& Chamorro-Premuzic, 2010). Os idosos, com frequência, podem sofrer com as doenças crônicas, as mortes dos amigos ou cônjuges e o isolamento social (Rokach \& Neto, 2005), mas podem continuar a ter relações amorosas satisfatórias. Neto (2012) mostrou que até a paixão erótica pode ser experimentada com intensidade por idosos. Isto sugere que a mesma pode estar presente em todo o ciclo da vida adulta.

Na velhice, é vivenciada a última crise, integridade versus desespero. A integridade, baseada na reflexão sobre a vida, é a capacidade de se preservar em todos os aspectos, de não se perceber acabado, confuso e desamparado. É necessário avaliar, recapitular e aceitar. A sabedoria significa, sem arrependimentos, aceitar o que viveu e que os outros merecem ser amados, mesmo com suas limitações (Erikson, 1998).

Nas últimas décadas, há um aumento global expressivo da expectativa de vida. Se as tendências dos últimos 23 anos se mantiverem, até 2030 a expectativa de vida global média das mulheres será 85,3 anos e a dos homens, 78,1 anos (The Lancet, 2015). No Brasil, a população de idosos chegará a $26,7 \%$ do total em 2060. A redução da natalidade e a evolução da medicina são alguns dos fatores que contribuem para este índice (Instituto Brasileiro de Geografia e Estatística, 2010). Consequentemente, o conhecimento sobre o amor e o envelhecimento é agora mais importante do que nunca.

Embora o amor seja importante para as pessoas de todas as idades, muitos pesquisadores que abordaram este tema por meio da Teoria Triangular do Amor (Sternberg, 1986) contemplaram em seus estudos a participação de jovens universitários com idade média em torno dos 25 anos (Acker \& Davis, 1992; Andrade, Garcia, \& CassepBorges, 2013; Cassep-Borges \& Pasquali, 2012; Cassep-Borges \& Teodoro, 2007, 2009; Gouveia, Fonseca, Cavalcanti, Diniz, \& Dória, 2009; Neto, 2001; Overbeek, Ha, Scholte, Kemp, \& Engels, 2007). Se o amor pode mudar ao longo do curso do relacionamento, a evidência disso precisa vir de respondentes representativos de toda a amplitude de idades e de tempos de duração das relações. Este foi o foco que a presente investigação explorou.

Sternberg (1986) desenvolveu a Teoria Triangular do Amor e sustentou que o amor é, principalmente, composto por intimidade, paixão e decisão/compromisso. Estes podem ser compreendidos pela metáfora de um triângulo, no qual cada componente ocupa um vértice deste. Cada amante tem um triângulo de amor resultante da combinação desses componentes. Existem vários tipos de amor, mas nem todos incluem todos os componentes ou exigem peso igual para os mesmos. 
A paixão é um estado de forte desejo de união com o outro, podendo ser considerada como o componente "quente" no qual estão os sentimentos mais intensos. A necessidade sexual pode ser condutora da paixão em várias relações amorosas, mas não a única, outras necessidades podem tomar parte, tais como as relacionadas à autoestima, à afiliação, à dominância/submissão e à autoatualização (Sternberg, 1986).

A intimidade se refere aos sentimentos de proximidade, o elo que une os amantes em dedicação e afeição comuns. Os sinais da intimidade são: o desejo de promover o bem-estar do objeto de amor; o experimentar felicidade ao estar com ele; o ter por ele alta consideração; poder contar com o ser amado em momentos de necessidade; a compreensão mútua; o dividir tanto a sua pessoa quanto as posses com o objeto de amor; o receber dele apoio emocional; o comunicar-se intimamente com este objeto; e o conceder-Ihe um valor importante. A intimidade é definida como um fundamento do amor que se desenvolve lentamente e resulta em conexões fortes entre as pessoas, que podem manter os relacionamentos (Sternberg, 1986).

O componente decisão/compromisso consiste de dois aspectos: a decisão de amar a outra pessoa, que é de curto prazo, e o compromisso de manter esse amor, que é de longo prazo. Estes não necessariamente andam juntos, a decisão de amar alguém pode não implicar num compromisso com esse amor. O compromisso é o componente mais premeditado do amor (Sternberg, 1986).

Sternberg (1986) sugeriu que cada um dos componentes do amor tem um curso diferente através do tempo de duração da relação amorosa. Os níveis da intimidade, paixão e compromisso podem mudar com o tempo e isso resultar numa alteração do relacionamento.

Normalmente, a paixão é mais forte no começo do relacionamento. Com o tempo e o sexo regular, ela se estabiliza numa forma menos urgente. A urgência poderá reaparecer em certos períodos, por exemplo, quando o casal fica separado por longo tempo, mas a falta dela não significa ausência de paixão. Para a compreensão do curso temporal da paixão, Sternberg (1986) recorreu à Teoria do Processo Oposto da Motivação Adquirida de Solomon (1980), a qual postula que o desejo por uma pessoa é função de dois processos subjacentes, positivo e negativo. A atração física e psicológica por outra pessoa pode levar ao surgimento da paixão. A passionalidade cresce rápido, mas, também, com rapidez alcança a expressão máxima. Quando atinge esse pico, uma força negativa começa a atuar e, literalmente, se opõe à paixão, que começa a decrescer e, gradualmente, um estado de habituação se estabelece, as forças positivas e negativas atingem o equilíbrio. Se a pessoa amada fosse perdida, haveria um retorno à linha de base ou ausência de passionalidade, mas é 
provável que a depressão e o mal-estar predominassem, devido à perda da força passional positiva (a pessoa desejada se foi) e à presença da força negativa (persiste o sentimento dos efeitos da ausência). Yela (1996, 2006), mediante Análise Fatorial, extraiu um modelo modificado da Teoria Triangular do Amor que chamou de Tetrangular, no qual a paixão foi subdividida em erótica e romântica. No início da relação, a intimidade aumenta continuamente. Depois, esse crescimento se torna mais lento e se estabiliza. No começo, os parceiros amorosos não se conhecem, há muita coisa para descobrir. A cada nova descoberta pode crescer a união e, com o tempo, os amantes podem tornarem-se mais previsíveis um para o outro e perceberem uma diminuição dos sentimentos mútuos. Isso pode indicar que estão se afastando, mas, também, pode significar que o relacionamento está se solidificando. No entanto, devido à estabilidade da relação, os parceiros podem não notar mais a interdependência (Sternberg, 1989).

As idéias de Sternberg (1989) sobre o curso temporal da intimidade se baseou na Teoria Geral das Emoções de Berscheid (1983), que se inspirou na Teoria Geral das Emoções de Mandler (1975). Conforme estas teorias, as emoções nas relações íntimas são experimentadas como resultado da interrupção das interações estereotipadas entre os parceiros. Uma ação esperada de um parceiro não causará emoção nenhuma no outro parceiro, mas, certamente, uma ação inesperada levará o outro a sentir alguma emoção. Quando duas pessoas se conhecem desenvolvem, gradativamente, uma série de scripts ou comportamentos estereotipados. A princípio, há incerteza, não se pode prever o comportamento do parceiro. Inevitavelmente, serão produzidas frequentes interrupções e rupturas na relação até que os dois se conheçam mais. Com o tempo, as interrupções diminuirão em frequência, os parceiros se tornarão mais previsíveis e mais dependentes entre si. Berscheid (1983) explicou que, à medida que diminuirão as interrupções, diminuirão as emoções, até não serem mais sentidas pelos parceiros. Nesta perspectiva, o amor pode ser entendido como um processo de redução da incerteza.

A diminuição da intimidade, em uma relação amorosa, tem aspectos positivo e negativo. O positivo, a diminuição da intimidade é resultado do aumento da vinculação interpessoal ou da aproximação do casal. Os parceiros estão tão conectados entre si que um não reconhece mais a presença do outro. Esta relação é possuidora de grande quantidade de intimidade oculta ou latente. $O$ negativo, a aparente falta de intimidade pode dificultar a discriminação entre a relação íntima e a ausência de intimidade. A maneira mais eficaz de distinguir a relação exitosa da fracassada é provocar alguma interrupção. A mudança da rotina ou o afastamento de um dos membros do casal por um tempo podem ser úteis para avaliar o grau da intimidade. Às vezes, somente com intervenções extremas é que 
se conhece a intimidade existente. Com frequência, casais que aparentam estar em crise, pois discutem e brigam, podem ter um grande grau de intimidade. Muitos casais que se separam não têm consciência do grau de intimidade investido na relação e, posteriormente, podem se arrepender. As pequenas interrupções podem ser positivas para promover a consciência do grau de intimidade latente, antes que se efetive a dissolução do amor (Sternberg, 1989).

Sternberg (1989) diz que a influência do tempo na intensidade do compromisso depende do sucesso do relacionamento. Seu nível é zero quando os dois amantes se conhecem. Geralmente o compromisso começa gradualmente, depois seu crescimento acelera. Se o relacionamento continua por um longo tempo, a sensação de compromisso se firma, tornando-se estável. Se o relacionamento fracassa, seu nível declina, podendo voltar à zero ou se transformar em outra forma de compromisso.

Vários estudos empíricos que utilizaram a Escala Triangular do Amor de Sternberg (1988) têm testado as predições da Teoria Triangular do Amor com relação a evolução temporal dos seus componentes. Acker e Davis (1992) realizaram uma pesquisa com 204 participantes de 18 a 68 anos de idade e tempo médio de duração da relação de 9,5 anos $(D P=10,0)$ que foram divididos em três grupos de tempos de duração da relação: até 3 anos, de 3 a 10 anos e acima de 10 anos. No geral, os resultados ofereceram apoio parcial para a teoria. Os respondentes mais velhos autorrelataram níveis mais baixos de paixão. Usando a variável tipo de relação (ou estágio da relação) como medida do curso temporal, o aumento do compromisso relatado emergiu muito claro, relacionamentos mais sérios foram associados com maior intimidade. No entanto, a intimidade relatada falhou em mostrar decréscimo ao longo do tempo de duração da relação. 0 declínio predito da intimidade foi encontrado apenas para os comportamentos de intimidade ao longo do tempo de duração da relação, por outro lado, o tipo de relação foi associado com aumento dos comportamentos de intimidade. O declínio previsto da paixão relatada ao longo do tempo de duração da relação emergiu apenas entre mulheres, contudo houve declínio para ambos os sexos em comportamentos de paixão nas relações mais longas.

A Teoria Triangular do Amor de Sternberg (1986) também foi avaliada por Serrano e Carreño (1993), que investigaram 114 casais com tempo médio de duração da relação de sete anos e quatro meses e idade média dos parceiros de 29 anos $(D P=6,92)$. Na intimidade, os resultados apuraram modificações relativas ao tempo de duração da relação, com declínio para ambos os membros dos casais. Da mesma forma, diminuiu a paixão para ambos os parceiros, mas o compromisso mostrou crescimento e, depois de certo tempo, estabilizou-se. 
Yela (1997) avaliou 412 universitários com idade média de 22,9 anos que estavam envolvidos em uma relação amorosa de diversos tipos em média há 2,9 anos $(D P=2,94)$. A maioria dos respondentes era solteira e não coabitava com os parceiros. Os respondentes foram classificados em sete faixas de tempos de duração da relação, que variaram de menos de dois meses até mais de sete anos. Nos resultados, o compromisso foi menos importante no princípio e, gradativamente, foi aumentando sua intensidade, mais rapidamente do que o esperado, até estabilizar-se em um nível de notável magnitude, aproximadamente no quarto ano. A evolução temporal da intimidade também foi bastante similar ao predito na teoria de Sternberg. Houve uma primeira etapa de crescimento rápido e continuado (até os quatro anos, aproximadamente) e uma segunda, de crescimento leve e estabilização. A curva temporal de paixão romântica encontrada também corroborou a previsão teórica. No princípio, a paixão romântica cresceu gradativamente e continuou aumentando progressivamente até muito depois da paixão erótica alcançar seu máximo. Com o tempo, o descenso da paixão romântica não alcançou valores tão baixos como os da paixão erótica. Curiosamente, a paixão erótica diminuiu mais entre os homens do que entre as mulheres.

Rocha e Hernandez (2002) compararam os escores de um grupo de 42 adultos jovens (de 18 a 25 anos de idade) e de um grupo de 18 idosos (com mais de 60 anos) que vivenciavam relações amorosas estáveis. Os resultados mostraram diferenças estatísticas significativas entre os grupos apenas no compromisso, sendo o nível deste mais elevado para os idosos. Os homens apresentaram níveis de paixão significativamente maiores do que as mulheres, entre os idosos.

No estudo de Villar, Villamizar e López-Chivrall (2005), 144 pessoas maiores do que 65 anos de idade $(M=73, D P=4,29)$ e com tempo médio de duração da relação maior do que 30 anos $(M=46,6, D P=$ $6,73)$ responderam à Escala Triangular do Amor de Sternberg (1988). A experiência amorosa relatada pelos idosos pareceu similar à dos jovens, mas não idêntica. Os níveis de compromisso apresentaram-se mais elevados do que os da paixão. As mulheres mostraram níveis inferiores de paixão na comparação com os homens.

Ahmetoglu, Swami e Chamorro-Premuzic (2010) analisaram os escores de 16.030 participantes, de 20 a 70 anos de idade, que responderam on line à Escala Triangular do Amor na versão reduzida adaptada por Lemieux e Hale (1999). Os resultados da modelagem de equações estruturais revelaram a idade dos participantes associada negativamente com a paixão e positivamente com intimidade e compromisso. Também o tempo de duração da relação foi negativamente associado com a paixão e positivamente com 0 compromisso. 
Toba (2010) investigou se as relações amorosas de 84 universitários (66 mulheres e dezoito homens) são tão vulneráveis aos efeitos do tempo, como as relações que ocorrem mais tarde na vida. A idade média dos participantes foi 21.96 anos $(D P=2,8)$ e o tempo médio de duração da relação foram 23,97 meses $(D P=21,50$ meses). Os resultados mostraram os escores de compromisso, intimidade e paixão significativamente correlacionados com o tempo de duração da relação. Contrário ao previsto, a paixão não diminuiu com o tempo.

Sumter, Valkenburg e Peter (2013) investigaram on line as percepções de amor ao longo do ciclo vital usando a Teoria Triangular do Amor. Os pesquisadores verificaram as diferenças de idade e sexo nos componentes do amor numa amostra de 12 a 88 anos de idade ( $n=2791$ ) dividida em faixas etárias. Adolescentes (12-17 anos) relataram níveis mais baixos em todos os componentes, comparados com adultos jovens (18-30 anos). Adultos com mais de 50 anos relataram os mais baixos níveis de paixão e intimidade, mas níveis similares de compromisso comparados com adultos jovens (18-30 anos) e adultos maduros (31-50 anos).

No presente estudo, foi realizada uma pesquisa exploratória transversal, comparando as medidas do amor em grupos de diferentes faixas etárias, classificados como adultos jovens, maduros e maiores de 50 anos. Desta forma, buscaram-se evidências da evolução dos componentes do amor ao longo do ciclo vital humano adulto.

\section{Método}

\subsection{Participantes}

Participaram deste estudo 160 pessoas do sexo feminino $(75,8 \%)$ e 51 do masculino (24,2\%), e a idade dos mesmos variou de 18 a 86 anos $(M=39,4, D P=19,78)$. Foram excluídos todos os indivíduos que apresentavam idade menor do que 18 anos. O tempo de duração da relação dos pesquisados variou de um a 63 anos $(M=14,3, D P=$ 14,86). Seguindo critérios semelhantes aos de Sumter et al. (2013), os sujeitos foram classificados em adultos jovens (18 a 30 anos de idade), adultos maduros ( 31 a 50 ) e maiores do que 50 anos. Do total de participantes, $88(41,7 \%)$ estavam namorando, oito $(3,8 \%)$ eram noivos, $85(40,3 \%)$ eram casados, $26(12,3 \%)$ estavam em união estável e quatro $(1,9 \%)$ estavam em outros tipos de relação amorosa. Considerando que alguns tipos de relação (noivado e união estável) apresentaram um número reduzido de participantes, os mesmos foram reagrupados em dois grupos: um, composto de namoro e noivado, e outro, de casamento e união estável (Tabela 1). Os sujeitos que declararam outros tipos de relação foram excluídos 
deste procedimento devido à dificuldade de classificá-los.

\begin{tabular}{|c|c|c|c|c|c|}
\hline Sexo & & $\begin{array}{l}(18-30 \\
\text { anos) }\end{array}$ & $\begin{array}{l}(31-50 \\
\text { anos) }\end{array}$ & $\begin{array}{l}\text { (> } \\
\text { anos) }\end{array}$ & 50Total \\
\hline \multirow{3}{*}{ Feminino } & Namoro e noivado & $\begin{array}{l}74 \\
(91,4 \%)\end{array}$ & $3(3,7 \%)$ & $4(4,9 \%)$ & 81 \\
\hline & $\begin{array}{l}\text { Casamento e união } \\
\text { estável }\end{array}$ & $\begin{array}{l}11 \\
(14,5 \%)\end{array}$ & \multirow{2}{*}{$\begin{array}{l}31 \\
(40,8 \%) \\
34 \\
(21,7 \%) \\
\end{array}$} & \multirow{2}{*}{$\begin{array}{l}34 \\
(44,7 \%) \\
38 \\
(24,2 \%)\end{array}$} & 76 \\
\hline & Total & $\begin{array}{l}85 \\
(54,1 \%)\end{array}$ & & & 157 \\
\hline \multirow{3}{*}{ Masculino } & Namoro e noivado & $\begin{array}{l}13 \\
(86,7 \%)\end{array}$ & $1(6,7 \%)$ & $1(6,7 \%)$ & 15 \\
\hline & $\begin{array}{l}\text { Casamento e união } \\
\text { estável }\end{array}$ & $2(5,7 \%)$ & $\begin{array}{l}11 \\
(31,4 \%)\end{array}$ & $\begin{array}{l}22 \\
(62,9 \%)\end{array}$ & 35 \\
\hline & Total & $15(30 \%)$ & $12(24 \%)$ & $23(46 \%)$ & 50 \\
\hline \multirow{3}{*}{ Total } & Namoro e noivado & $\begin{array}{l}87 \\
(90,6 \%)\end{array}$ & $4(4,2 \%)$ & $5(5,2 \%)$ & 96 \\
\hline & $\begin{array}{l}\text { Casamento e união } \\
\text { estável }\end{array}$ & $\begin{array}{l}13 \\
(11,7 \%)\end{array}$ & $\begin{array}{l}42 \\
(37,8 \%)\end{array}$ & $\begin{array}{l}56 \\
(50,5 \%)\end{array}$ & 111 \\
\hline & Total & $\begin{array}{l}100 \\
(48,3 \%)\end{array}$ & $\begin{array}{l}46 \\
(22,2 \%)\end{array}$ & $\begin{array}{l}61 \\
(29,5 \%)\end{array}$ & 207 \\
\hline
\end{tabular}

\subsection{Instrumentos}

Foi utilizada a Escala Triangular do Amor de Sternberg (1997, 1998) na adaptação de Hernandez (1999). Este instrumento é composto de 45 itens, igualmente divididos entre três componentes: intimidade, paixão e decisão/compromisso. Para responder aos itens foi usada uma escala tipo Likert de cinco pontos: 1 "nada verdadeiro" e 5 "totalmente verdadeiro". Os participantes também forneceram as seguintes informações: idade, sexo, tipo e tempo de duração da relação.

\subsection{Coleta de dados}

A coleta dos dados foi realizada no campus universitário principal da Universidade do Estado do Rio de Janeiro (UERJ) com estudantes de diversos cursos. Os idosos, na maioria, eram alunos da Universidade Aberta da Terceira Idade (UNATI-UERJ). Contudo, uma parte dos dados foi obtida em grupos de amigos, clínicas médicas, academias de ginástica, parques e praças. Os dados foram coletados de ambas as formas, em grupos e individualmente.

\subsection{Procedimentos relativos à Ética}


A presente pesquisa foi aprovada através do parecer no 129.870 de $14 / 11 / 2012$ da universidade a qual está vinculada e observou os procedimentos relativos à ética da pesquisa com humanos. Todos os participantes da pesquisa leram e assinaram um Termo de Consentimento Livre e Esclarecido (TCLE).

\subsection{Análise de dados}

Para digitação e análise dos dados foi utilizado o SPSS versão 20, através de técnicas estatísticas descritivas e Análise Multivariada de Variância (MANOVA).

\section{Resultados}

Foi realizada uma MANOVA para os componentes do amor (paixão, intimidade e compromisso), incluídos como variáveis dependentes, e faixas etárias (adultos jovens, maduros e maiores de 50 ) e sexo (homens e mulheres) foram incluídos como variáveis independentes. A variável tipo de relação não foi incluída entre as independentes deste delineamento porque, em algumas condições das faixas etárias, - número de participantes ficou muito pequeno (Tabela 1). Estatísticas multivariadas foram significativas para Componentes do Amor, $\lambda$ de Wilks $=0,09, F(3,203)=3.663,10, p<0,001, n_{\mathrm{p}}{ }^{2}=$ 0,98 ; Componentes do Amor $X$ Faixa Etária, $\lambda$ de Wilks $=0,92, F(6$, 406) $=2,69, p<0,01, \eta_{p}{ }^{2}=0,04$; Componentes do Amor $X$ Sexo, $\lambda$ de Wilks $=0,95, F(3,203)=3,50, p<0,01, \eta_{p}{ }^{2}=0,05$. A interação Componentes do Amor X Faixa Etária X Sexo não foi significativa.

Tabela 2

Níveis Médios e Desvios Padrão dos Componentes do Amor por Faixa Etária e Sexo

\begin{tabular}{llllllll}
\hline & & \multicolumn{2}{l}{ Homens } & \multicolumn{2}{l}{ Mulheres } & \multicolumn{2}{l}{ Todos } \\
\cline { 3 - 7 } Amostra total & & Paixão & In & $M$ & $D P$ & $M$ & $D P$ \\
\hline \multirow{4}{*}{ (18-30 anos) } & Intimidade & 4,60 & 0,62 & 3,94 & 0,74 & 3,99 & 0,72 \\
& Compromisso & 4,71 & 0,36 & 4,46 & 0,58 & 4,50 & 0,54 \\
& Paixão & 4,29 & 0,52 & 4,07 & 0,50 & 4,11 & 0,50 \\
& Intimidade & 4,66 & 0,25 & 4,61 & 0,32 & 4,62 & 0,31 \\
& Compromisso & 4,68 & 0,39 & 4,54 & 0,39 & 4,56 & 0,39 \\
(31-50 anos) & Paixão & 4,29 & 0,52 & 4,07 & 0,50 & 3,69 & 0,84 \\
& Intimidade & 4,58 & 0,43 & 4,10 & 0,79 & 4,21 & 0,74 \\
& Compromisso & 4,83 & 0,18 & 4,19 & 0,90 & 4,35 & 0,83 \\
& Paixão & 4,12 & 0,50 & 3,55 & 0,89 & 4,06 & 0,85 \\
(> 50 anos) & Intimidade & 4,58 & 0,44 & 4,50 & 0,55 & 4,53 & 0,58 \\
& Compromisso & 4,67 & 0,41 & 4,56 & 0,68 & 4,60 & 0,58 \\
\hline
\end{tabular}


As interações Componentes do Amor X Faixa Etária e Componentes do Amor X Sexo foram significativas. Como o objetivo principal foi estudar os padrões do amor ao longo da vida adulta, foi conduzida uma análise de follow-up para os três componentes separadamente. As análises univariadas incluíram faixa etária e sexo como variáveis independentes (Tabela 2).

$\mathrm{Na}$ variável dependente paixão não foram encontrados efeitos significativos para faixa etária, nem para a interação faixa etária $X$ sexo. Porém, um efeito principal foi observado para sexo, $F(1,205)=$ 6.792, $p<0,01, \eta_{p}{ }^{2}=0,032$. A média do grupo masculino $(4,2)$ foi maior do que a média do grupo feminino $(3,9)$.

$\mathrm{Na}$ variável dependente intimidade, um efeito principal foi observado para faixa etária (Figura 1), $F(2,205)=4.103, p=0,04, \eta_{p}^{2}=$ 0,091 . O teste de follow up de Bonferroni identificou que as diferenças significativas estão entre as médias dos grupos 31-50 anos $(3,7)$ e as médias dos grupos $18-30$ anos $(4,6)$ e $>50$ anos $(4,53)$. Não foi identificada diferença entre os grupos $18-30$ anos e $>50$ anos. Não foi encontrado efeito significativo para a interação faixa etária $X$ sexo. Um efeito principal significativo foi observado para sexo, $F(1$, $205)=5.436, p=0,021, \eta_{p}^{2}=0,026$. A média do grupo masculino $(4,6)$ foi maior do que a média do grupo feminino $(4,5)$.

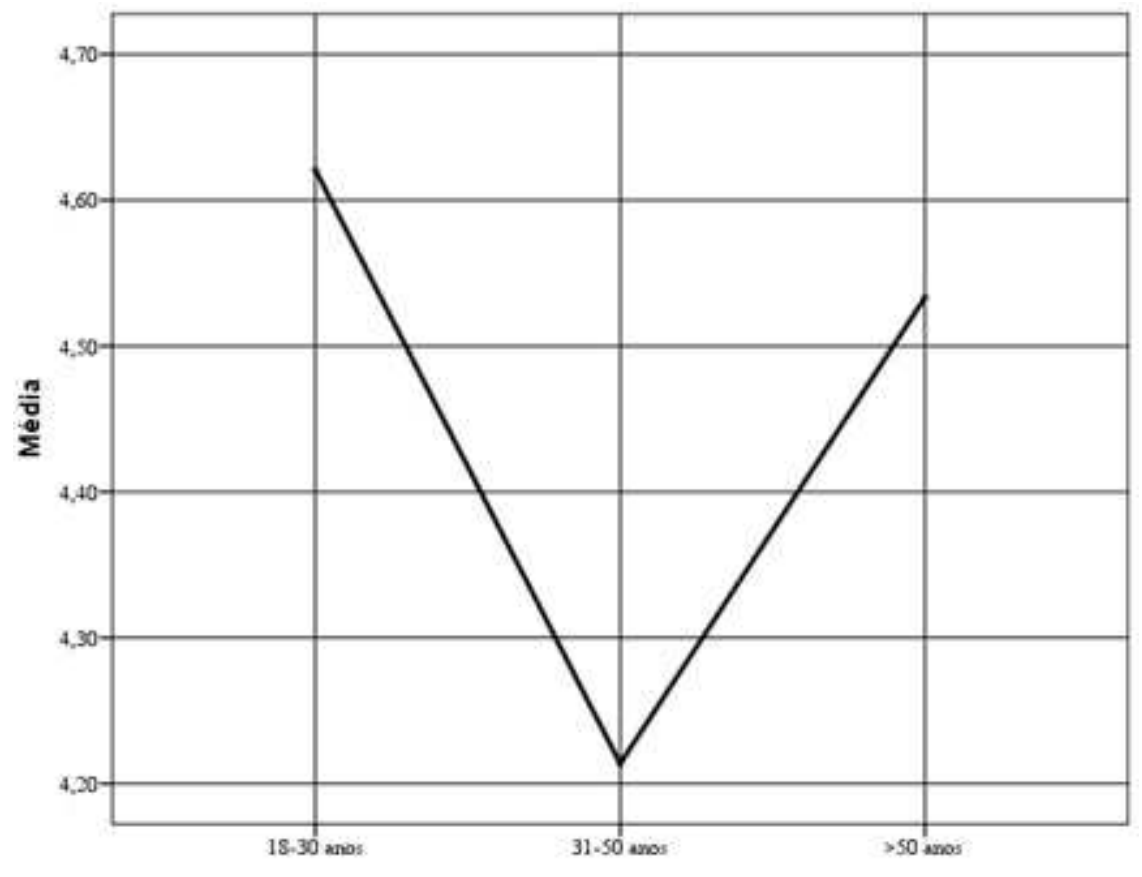

Figura 1

Médias de intimidade por faixa etária.

$\mathrm{Na}$ variável dependente compromisso, um efeito principal foi observado para sexo, $F(1,205)=9.556, p=0,002, \eta_{p}{ }^{2}=0,045$. A média do grupo masculino $(4,7)$ foi maior do que a média do grupo feminino $(4,5)$. Não foram encontrados efeitos significativos para 
faixa etária e para a interação faixa etária X sexo.

Discussão

O presente estudo procurou identificar evidências da evolução temporal do amor por meio de um delineamento transversal comparando três grupos de faixas etárias de indivíduos envolvidos em algum tipo de relação amorosa. No entanto, apenas o componente intimidade apresentou uma variação significativa ao longo das faixas etárias. Os níveis de intimidade foram mais elevados para o grupo de 18-30 anos, sofreram uma queda significativa para o grupo de 31-50 anos e se recuperaram para o grupo dos $>$ de 50 anos. Conforme a Teoria Triangular do Amor de Sternberg (1998), a explicação para este declínio da intimidade dos adultos jovens para os maduros está na Teoria da Geral das Emoções de Berscheid (1983). O decréscimo da intimidade (Figura 1) representaria uma redução da intimidade manifesta (percebida), mas não da intimidade latente (não percebida). A intimidade tende a aumentar com o tempo de relação, os indivíduos do grupo de 18-30 anos apresentaram um tempo médio de relação de três anos e seis meses, espaço temporal ótimo para que a intimidade possa atingir patamares elevados. Com a passagem do tempo, a média de duração de relação do grupo de 3150 anos foi de 15 anos, os parceiros estão tão conectados entre si que um não reconhece mais a presença do outro. A diminuição da intimidade, neste caso, pode ser resultado do aumento da vinculação interpessoal e da aproximação do casal. Embora a medida da intimidade percebida apresente graus menores, esta relação pode possuir grande quantidade de intimidade oculta ou latente (Sternberg, 1998). Concomitantemente, os investimentos que os parceiros amorosos dedicam às suas carreiras profissionais e aos cuidados com os filhos também podem explicar, em parte, esse "esquecimento" da intimidade (Smetana et al., 2006). No grupo de maiores de 50 anos (tempo médio de duração da relação de 30 anos), a cessação dessas demandas e a consequente retomada da atenção dos parceiros à vida conjugal levariam à recuperação da intimidade manifesta (Sternberg, 1998), o que explicaria os elevados níveis medidos na pesquisa atual. No entanto, o tamanho do efeito das diferenças entre os grupos de faixa etária avaliados foi pequeno, revelando que $9 \%$ da variação da intimidade pode ser explicada pela variável faixa etária.

Além disso, os resultados revelaram variações significativas nos três componentes do amor (paixão, intimidade e compromisso) devido ao sexo dos participantes. Em todos os casos, os componentes do amor apresentaram níveis mais elevados para os homens. Contudo, todas as diferenças de sexo relativas aos níveis de paixão, intimidade e compromisso deste estudo foram de pequeno tamanho, 3\%, 3\% e $4 \%$, respectivamente.

De acordo com os estereótipos prevalece que os homens são mais 
relutantes em assumir compromissos e, muitas vezes, procuram evitar a intimidade. Mas os dados mostram que os homens se apaixonam mais facilmente que as mulheres, têm maior reatividade emocional do que as mulheres. Assim, os homens apresentam mais paixão e um crescimento menor de intimidade do que as mulheres (Baumeister \& Bratslavsky, 1999). Nas pesquisas de Hyde (2005) e Ahmetoglu et al. (2010), os homens também apresentaram níveis mais elevados de paixão do que as mulheres.

$\mathrm{Na}$ literatura PSI, com relação ao componente compromisso, os resultados de diferenças sexuais têm sido inconsistentes. Algumas pesquisas não encontram diferenças significativas (Gao, 2001; Hyde, 2005), outras relatam que os homens apresentaram mais compromisso do que as mulheres (Ahmetoglu et al., 2010) ou que mulheres relatam mais compromisso do que homens (Lemieux \& Hale, 1999).

No geral, alguns estudos têm encontrado diferenças entre homens e mulheres nas medidas dos componentes do amor (Acker \& Davis, 1992; Hendrick \& Hendrick, 1986; Rocha \& Hernandez, 2002; Sumter, Valkenburg, \& Peter, 2013; Villar, Villamizar, \& LópezChivrall, 2005), mas outras pesquisas não (Ahmetoglu et al., 2010; Serrano \& Carreño, 1993; Sternberg, 1997) Os resultados controversos parecem corroborar a idéia de que as abordagens convencionais sobre diferenças sexuais e amor têm recebido apenas apoio limitado da pesquisa em ciências sociais e comportamentais.

Nos estudos mais recentes, as diferenças sexuais na percepção do amor parecem menos robustas do que antes (Gao, 2001; $\mathrm{Ha}$, Overbeek, de Greef, Scholte, \& Engels, 2010, Hyde, 2005). Alguns pesquisadores defenderam a idéia que a experiência do amor depende mais da orientação de gênero do que do sexo dos participantes (Coleman \& Ganong, 1985) e que as diferenças no amor dentre os sexos são maiores do que entre os sexos (Sumter et al., 2013). As pequenas e inconsistentes evidências de diferenças de sexo que têm sido encontradas na pesquisa do amor podem ser reflexo da gradual diminuição geral das diferenças sexuais na sociedade atual. As descobertas de Schoenfeld, Bredow e Huston (2012) indicaram que homens e mulheres apresentaram em seus amores mais nuances do que os estereótipos culturais sugerem.

O presente estudo teve como objetivo central compreender a evolução do amor através da comparação de quatro fases do desenvolvimento humano: jovens, adultos jovens, maduros e idosos. Também foram buscadas correlações entre os componentes do amor, compromisso, intimidade e paixão com a idade, faixas etárias, tempo de relação, sexo e tipo ou estágio de relação dos participantes. Nos resultados, houve apenas apoio parcial para o desenvolvimento do curso temporal dos componentes do amor previsto na Teoria Triangular do Amor. 
Muito embora tenham aparecido evidências que corroboram o modelo teórico, em alguns estudos anteriores, em outros, isso não ocorreu. De modo geral, os tamanhos e, principalmente, as características das amostras (idade dos participantes e tempo de duração da relação) e procedimentos, têm diferido entre os estudos, o que poderia explicar os diferentes resultados.

Uma amostra restrita a região metropolitana do Rio de Janeiro, de tamanho moderado e não probabilística, como a usada neste estudo, pode fornecer dados enviesados e resultados que não refletem a realidade e os modelos teóricos. Sugere-se que novas investidas sejam feitas com amostras mais abrangentes e o uso de uma estratégia longitudinal, que seria mais consistente para a investigação da evolução temporal do amor. Também se recomenda que, em futuras pesquisas sobre relações amorosas, além do sexo, seja incluída a variável orientação ou identidade de gênero dos participantes.

\section{Referências}

Acker, M., \& Davis, M. H. (1992). Intimacy, Passion and Commitment in Adult Romantic Relationships: A Test of the Triangular Theory of Love. Journal of Social and Personal Relationships, 9, 21-50.

Andrade, A. L., Garcia, A., \& Cassep-Borges, V. (2013). Evidências de validade da Escala Triangular do Amor de Sternberg - Reduzida (ETAS-R). Psico-USF, 18, 501-510.

Ahmetoglu, G., Swami, V., \& Chamorro-Premuzic, T. (2010). The Relationship Between Dimensions of Love, Personality, and Relationship Length. Archives of sexual behavior, 39, 11811190.

Arnett, J. J. (2000). Emerging adulthood: a theory of development from the late teens through the twenties. American Psychologist, 55, 469-80.

Baumeister, R. F., \& Bratslavsky, E. (1999). Passion, Intimacy, and Time: Passionate Love as a Function of Change in Intimacy. Personality and Social Psychology Review, 3, 49-67.

Berscheid, E. (1983). Emotion. In: H. H. Kelley, E. Berscheid, A. Christensen, J. H. Harvey, T. L. Huston, G. Levinger, E. McClintock, L. A. Peplau, \& D. R. Peterson(Eds). Close Relationships (pp. 110-168). New York: Freeman.

Cassep-Borges, V., \& Pasquali, L. (2012). Estudo Nacional dos Atributos Psicométricos da Escala Triangular do Amor de Sternberg. Paidéia, 22, 21-31.

Cassep-Borges, V., \& Teodoro, M. L. M. (2007). Propriedades Psicométricas da Versão Brasileira da Escala Triangular do Amor de Sternberg. Psicologia: Reflexão e Crítica, 20, 513-522. 
Cassep-Borges, V., \& Teodoro, M. L. M. (2009). Versión Reducida de la Escala Triangular del Amor: Características del Sentimiento en Brasil. Revista Interamericana de Psicología, 43, 30-38.

Coleman, M., \& Ganong, L. H. (1985). Love and Sex Role Stereotypes: Do Macho Men and Feminine Women Make Better Lovers? Journal of Personality and Social Psychology, 49, 170176.

Erikson, E. (1998). O ciclo de vida completo. Porto Alegre: Artes Médicas.

Gao, G. (2001). Intimacy, passion and commitment in Chinese and US American romantic relationships. International Journal of Intercultural Relations, 25, 329-342.

Gouveia, V. V., Fonseca, P. N., Cavalcanti, J. P. N., Diniz, P. K. C., \& Dória, L. C. (2009). Versão abreviada da Escala Triangular do Amor: evidências de validade fatorial e consistência interna. Estudos de Psicologia, 14, 31-39.

Ha, T., Overbeek, G., de Greef, M., Scholte, R. H. J., \& Engels, R. C. M. E. (2010). The importance of relationships with parents and best friends for adolescents' romantic relationship quality: Differences between indigenous and ethnic Dutch adolescents. International Journal of Behavioral Development, 34, 121-127.

Hendrick, C., \& Hendrick, S. S. (1986). A theory and method of love. Journal Personality and Social Psychology, 50, 392-402.

Hernandez, J. A. E. (1999). Validação da estrutura da Escala Triangular do Amor: análise fatorial confirmatória. Aletheia, 9, $15-25$.

Hyde, J. S. (2005). The gender similarities hypothesis. American Psychologist, 60, 581-592.

Instituto Brasileiro de Geografia e Estatística (2010). Censo 2010. Recuperado em 15 de novembro, 2013, de: http://www.censo2010.ibge.gov.br/resultados

Lemieux, R., \& Hale, J. L. (1999). Intimacy, Passion, and Commitment in Young Romantic Relationships: Successfully Measuring the Triangular Theory of Love. Psychological Reports, 85, 497-503.

Mandler, G. (1975). Mind and Emotion. New York: Wiley.

Neto, F. (2001). Love Styles of Three Generations of Women. Marriage \& Family Review, 33, 19-30.

Neto, F. (2012). Perceptions of love and sex across the adult life span. Journal of Social and Personal Relationships, 29, 760775.

Overbeek, G., Ha, T., Scholte, R., Kemp, R., \& Engels, R. C. M. E. (2007). Brief report: Intimacy, passion, and commitment in romantic relationships-Validation of a 'triangular love scale' for adolescents. Journal of Adolescence, 30, 523-528.

Rocha, E., \& Hernandez, J. A. E. (2002). Os jovens, os idosos e o 
Amor. In: XIV Salão de Iniciação Cientifica e XI Feira de Iniciação Científica, Universidade Federal do Rio Grande do Sul. Livro de Resumos. Porto Alegre: Gráfica da UFRGS, 802-802.

Rokach, A., \& Neto, F. (2005). Age, culture, and the antecedents of loneliness. Social Behavior and Personality: an international journal, 33, 477-494.

Schoenfeld, E. A., Bredow, C. A., \& Huston, T. L. (2012). Do Men and Women Show Love Differently in Marriage? Personality and Social Psychology Bulletin, 38, 1396-1409.

Serrano, G. M., \& Carreño, M. F. (1993). La teoria de Sternberg sobre el amor. Analisis empirico. Psicothema, 5, 151-167.

Smetana, J. G., Campione-Barr, N., \& Metzger, A. (2006). Adolescent Development in Interpersonal and Societal Contexts. Annual Review of Psychology, 57, 255-284.

Solomon, R. (1980). The opponent Process Theory of Acquired Motivation: The Cost of Pleasure and the Benefits of Pain. American Psychologist, 35, 691-712.

Sternberg, R. (1986). A Triangular Theory of Love. Psychological Review, 93, 119-135.

Sternberg, R. J., \& Barnes, M. L. (1988). The psychology of love. London: Yale University Press.

Sternberg, R. J. (1989). El triángulo del amor: intimidad, pasyon y compromisso. Barcelona: Paidós Ibérica.

Sternberg, R. J. (1997). Construct validation of a triangular love scale. European Journal of Social Psychology, 27, 313-335.

Sternberg, R. J. (1998). Cupid's Arrow: The course of love through time. Cambridge: University Press.

Sumter, S. R., Valkenburg, P. M., \& Peter, J. (2013). Perceptions of love across the lifespan: Differences in passion, intimacy, and commitment. International Journal of Behavioral Development, $37,417-427$.

The Lancet (2015). Global, regional, and national age-sex specific allcause and cause-specific mortality for 240 causes of death, 1990-2013: a systematic analysis for the Global Burden of Disease Study 2013. The Lancet, 385, 117-171.

Toba, J. (2010). Four Years, One Try (angle): Using Sternberg's Triangular Love Theory to Evaluate the Effects of Time on College Relationships [monograph].Recuperado em 08 de outubro, 2013, de: http://hdl.handle.net/10125/29675

Villar, F., Villamizar, D. J., \& López-Chivrall, S. (2005). Los componentes de la experiencia amorosa en la vejez: personas mayores y relaciones de pareja de larga duración. Revista Española de Geriatría y Gerontología, 40, 166-177.

Yela, C. G. (1996). Componentes básicos del amor: algunas matizaciones al modelo de Sternberg. Revista de Psicologia Social, 11, 185-201. 
Yela, C. G. (1997). Curso temporal de los componentes básicos del amor a lo largo de la relación de pareja. Psicothema, 9, 1-15.

Yela, C. (2006). The evaluation of love simplified version of the Scales for Yela's Tetrangular model basead on Sternberg's model. European Journal of Psychological Assessment, 22, 2127.

\section{Endereço para correspondência \\ José Augusto Evangelho Hernandez}

Universidade do Estado do Rio de Janeiro

Instituto de Psicologia

Rua São Francisco Xavier, 524, $10^{\circ}$ andar, Maracanã, CEP 20550-013, Rio de Janeiro - RJ, Brasil

Endereço eletrônico: hernandez.uerj@gmail.com

\section{Sinele Valle da Costa}

Universidade do Estado do Rio de Janeiro

Instituto de Psicologia

Rua São Francisco Xavier, 524, $10^{\circ}$ andar, Maracanã, CEP 20550-013, Rio de Janeiro - RJ, Brasil

Endereço eletrônico: sinellevc@hotmail.com

\section{Juliana Ramos Ribeiro}

Universidade do Estado do Rio de Janeiro

Instituto de Psicologia

Rua São Francisco Xavier, 524, $10^{\circ}$ andar, Maracanã, CEP 20550-013, Rio de Janeiro - RJ, Brasil

Endereço eletrônico: julianaramos_psi@hotmail.com

\section{Caroline Almeida Areias}

Universidade do Estado do Rio de Janeiro

Instituto de Psicologia

Rua São Francisco Xavier, 524, $10^{\circ}$ andar, Maracanã, CEP 20550-013, Rio de Janeiro - RJ, Brasil

Endereço eletrônico: line_1007@yahoo.com.br

\section{Karina Nascimento Valladares dos Santos}

Universidade do Estado do Rio de Janeiro

Instituto de Psicologia

Rua São Francisco Xavier, 524, $10^{\circ}$ andar, Maracanã, CEP 20550-013, Rio de Janeiro - RJ, Brasil

Endereço eletrônico: karinanvs91@yahoo.com.br

Recebido em: 31/05/2014

Reformulado em: 23/02/2015

Aceito para publicação em: 16/05/2015

\section{Notas}

* Doutor em Psicologia do Desenvolvimento (UFRGS). Professor Adjunto do Instituto de Psicologia da UERJ.

** Acadêmica do $8^{\circ}$ período do curso de Psicologia da UERJ.

*** Acadêmica do $8^{\circ}$ período do curso de Psicologia da UERJ.

**** Acadêmica do $8^{\circ}$ período do curso de Psicologia da UERJ.

$* * * * *$ Acadêmica do $8^{\circ}$ período do curso de Psicologia da UERJ. 